\title{
Notes on Dates and Transliteration
}

All dates for events in the narrative, unless otherwise specified, are in $\mathrm{AD}$ style and use numbers only, without the AD designation - for example, 1055. If there is any chance for ambiguity, then "BC" or "AH" (referring to the Muslim calendar, hijra dates) is used. If two dates are given for the same event, they are in the form $\mathrm{AH} / \mathrm{AD}$, but without the designations $\mathrm{AH}$ and $\mathrm{AD}$-for example, 140/757-758. Dates for sources are given only as AD dates.

Transliterations of Arabic words into Latin script are simplified. For example, the ta marbuta, the terminal Arabic silent $t$ rendered as an $b$ in English, is generally not used here. Initial hamzas (') and 'ayns (') are omitted, as are dots below and above consonants, and macrons above long vowels. Words that are commonly seen in English, such as "Quran" and "vizier," appear in that form and are not italicized. Words that often appear in French, such as oued rather than "wadi," are kept in French. Names of recent authors are written as the authors render their own names in Latin script. Names of places and persons that are still used today are written as they are in the country where they are located, for example, Meknes rather than Maknaas, Marrakech rather than Marrakush. Foreign words that are part of proper names are not italicized. In endnotes and in the bibliography, the rules for uppercase and lowercase in English are applied to all titles. 
THIS PAGE INTENTIONALLY LEFT BLANK 